\title{
Density Cum Priority based Adaptive Traffic Light Control System
}

\author{
A. Sahaya Ponrekha, Kamesh Rath, Shubham Ghosh, Utkarsh Dwivedi
}

\begin{abstract}
Advancement of technology and increase in population density expands the quantity of vehicles out. This in turn increases the traffic density, which necessitates the well developed traffic controller. This paper explains a microcontroller based traffic control system. To explain about the system a four way junction is considered. In addition to the normal traffic control system, two special cases are also handled in this system. The developed system is dealing problem of highly dense lane.IR sensors are used to detect the density of the vehicles. This system also help to avoid the unnecessary delay for the priority vehicles. RFID technique is used to identify the priority vehicles. An IOT WIFI module is used in this system to display the traffic signal status and to store the traffic data. This traffic data can be processed in future to design the improved adaptive control system
\end{abstract}

Keywords- IR sensor, RFID, Arduino UNO micro controller, IOT.

\section{INTRODUCTION}

With advancements in automated locomotion there has been a rift in the development of two prime components that provides for a complete automation of the road traffic. With major companies focused on making the vehicles autonomous, a gap has appeared in presenting similar effort in working from the side of the road infrastructure and heavy scale investments therein. Nowadays the traffic detection using GPS reflected signals is also proposed [1][2]. But if the travelers or drivers do not have GPS (Global Positioning system ) with them, this technique cannot be used. Many other methods need proper modeling of lanes and strategies are used to find the traffic density [3] .Traffic control using artificial intelligent algorithms are also proposed[4].But deriving algorithms and its implementation makes the system complex. The images obtained by the camera, which is fitted in to the controller are processed to control the traffic[5][6]. Some times image processing makes the traffic control system as costly. In reference[7] priority based traffic control is done by using an RF transmitter in priority vehicle. So this method[7] makes the necessity of fitting RF transmitter in all priority vehicle and also density based priority is not considered. The traffic control system with RFID technique

Revised Version Manuscript Received on 10, September 2019.

Mrs. A. Sahaya Ponrekha, Assistant Professor,Electrical and Electronics and Engineering Dept, SRM Institute of Science and Technology, Chennai, Tamil Nadu, India.(Email: sahayaponrekha.a@ktr.srmuniv.ac.in)

Kamesh Rath, Student,Electrical and Electronics and Engineering Dept, SRM Institute of Science and Technology, Chennai, Tamil Nadu, India.

Shubham Ghosh, Student, Electrical and Electronics and Engineering Dept, SRM Institute of Science and Technology, Chennai, Tamil Nadu, India.

Utkarsh Dwivedi, Student ,Electrical and Electronics and Engineering Dept, SRM Institute of Science and Technology, Chennai, Tamil Nadu, India. is also proposed[8], but in the system same RFID technique is used for congestion management also which can not count the vehicle which don't have RFID enable devices. In reference[9] emergency vehicle identification is done with a help of an antenna which is to be built in the vehicle. In this paper the system extrapolates input from the primary IR sensors that are fed into a microcontroller based device which in turn administer the switching of traffic lights. Hardware pertaining to this conglomeration include basic array of 8 IR sensors, Arduino UNO microcontroller, unique RFID tag specifications, LED lights and a software user interface to feed desirable commands. Primary IR sensors detect the density of the vehicular traffic perpetrating trough each of the four lanes. This feedback is assimilated by the Arduino UNO and allows for decongestion of a particular lane by allocating them additional time till the vehicular density coincides with a standard fed value. This operational method is proposed in the ref [2][11]. However it falls short of interpreting priority vehicle from among the masses of traffic[12]. Prior solution proposed in this issue includes manual operation of monitoring the proposed list of important vehicles and signalling the traffic lights[10][13]. This however obstructs the robustness of the control system and can be better achieved through automation. A developed system of RFID tagging helps for the detection of priority vehicles like ambulances and pursuit police cars, allowing them exclusive passage through the required lanes. Apart from being just a control system this facilitates the accumulation of traffic data in useful amounts, the potential benefits of surveying which are innumerable for further studies in safety and control of traffic systems. This traffic data can be accessed using the IOT WiFi module, which is used in this system.

\section{BLOCK DIAGRAM OF THE SYSTEM}

The figure 1 shows the block diagram of the system. Table 1 indicates the major hardware and software components required for the traffic control system. This traffic control system is applied for a four way junction. So eight LM358 IC ,IR sensors are used which helps to find the traffic density. EM-18 RFID reader module is used to read the RFID tag ,which is on the priority vehicle. The signal from the IR sensors and the RFID tag is processed using Arduino micro controller. The micro controller controls the LED and give signal to the EM-18 IOT WiFi module. 
Table 1.Hardware and software components required

\begin{tabular}{|c|c|c|}
\hline \multicolumn{2}{|c|}{ Hardware Requirements } & Software \\
\hline Component & $\begin{array}{l}\text { Model } \\
\text { Specification }\end{array}$ & \multirow[b]{2}{*}{ Arduino IDE } \\
\hline IR sensors & LM358 IC & \\
\hline $\begin{array}{ll}\text { RFID } & \text { reader } \\
\text { module } & \end{array}$ & EM-18 & \multirow{3}{*}{ Embedded C } \\
\hline micro controller & Arduino- UNO & \\
\hline LED & Red and Green & \\
\hline
\end{tabular}

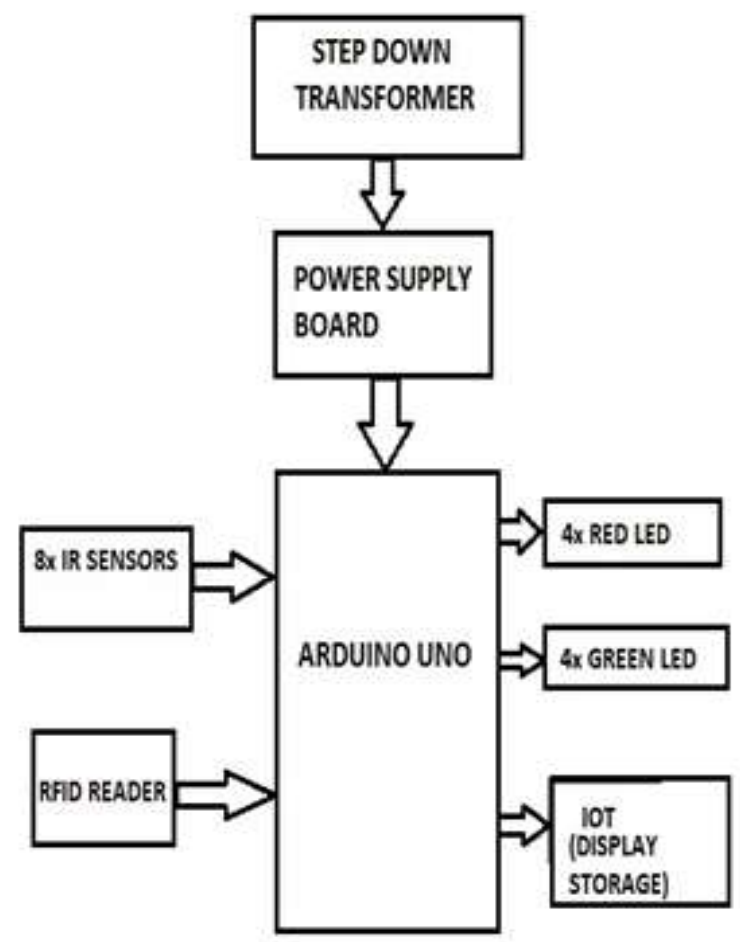

Fig.1.Block diagram of the system

\section{i)LM358 IC IR sensor Module}

This module has Infra Red transmitting tube which transmit IR wave continuously. When this wave hits on obstacle, the wave is reflected and will be received by the receiver tube in the module. The module has an in built comparator which is processing the signals and changes the output. The out pin goes low whenever the reflected signal is received. The overall structure of the IR sensor is shown in figure 2 .

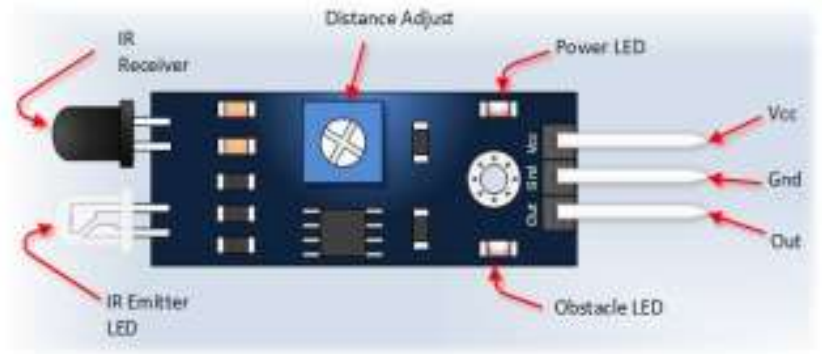

Fig.2. Structure of LM358 IC IR sensor Module

\section{ii)EM-18 RF reader module}

The EM-RF reader module can be used to read a RF tag with a frequency $125 \mathrm{kHz}$. It has an antenna which can be connected to the PC using RS232. The coil in this module radiates $125 \mathrm{kHz}$. When the RFID tag is coming towards the reader, the ID is read. The module sends the information to the Arduino micro controller. The controller receives the signal from the reader and process. The EM-18 is a nine pin module which is shown in figure 3 . The figure 4 shows the picture of EM-18 RF reader module.

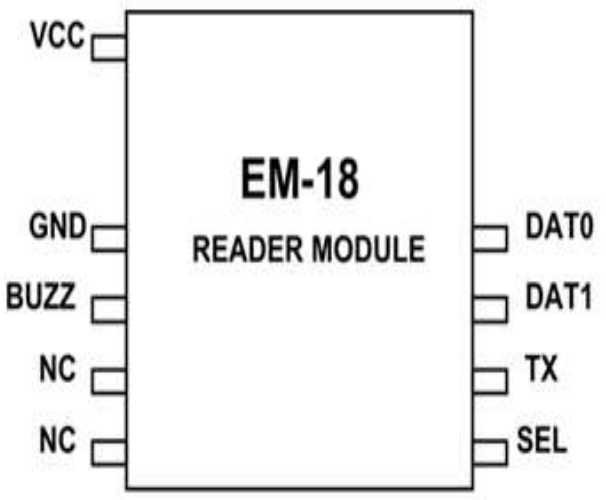

Fig.3.Pin diagram of EM-18 reader module

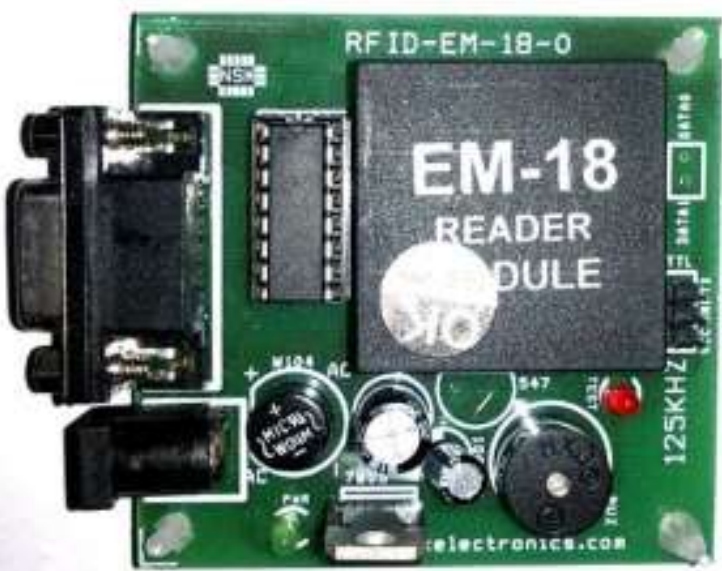

Fig.4. Picture of EM-18 reader module

iii) ESP8266 WiFi Module

This module has an incorporated TCP/IP protocol stack. The WiFi network can access the Arduino micro controller using this module. This module can host an application. To create a new application different types of firm wares can be loaded. To use for custom firmware, an $80 \mathrm{MHz}, 32$ bit processor is inbuilt in this module. Small webpage can be hosted using this module. The RF in this module helps to work this module in different operating conditions. The main advantage of this module is its low cost. The picture of the ESP8266 WiFi Module and its internal block diagram are shown in figure 5,6 respectively. 


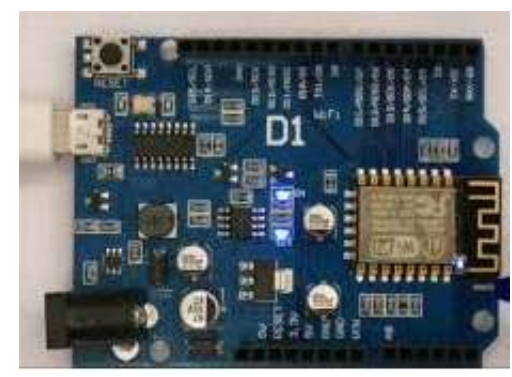

Fig.5. picture of ESP8266 IOT WiFi module

\section{iv)Arduino UNO micro controller}

It is board which is having ATmega328P microcontroller. It has $16 \mathrm{MHz}$ quartz crystal oscillator, 6 analog input pins and 14 digital input/output pins. Out of the 14 digital I/O, pins 6 pins can be utilized for PWM outputs. Every pins are operating with the voltage of $5 \mathrm{~V}$. Arduino can be connected to other microcontrollers and computers also. Arduino has to be configured with Arduino IDE software. Figure 7 shows the picture of Arduino UNO board with its specifications.

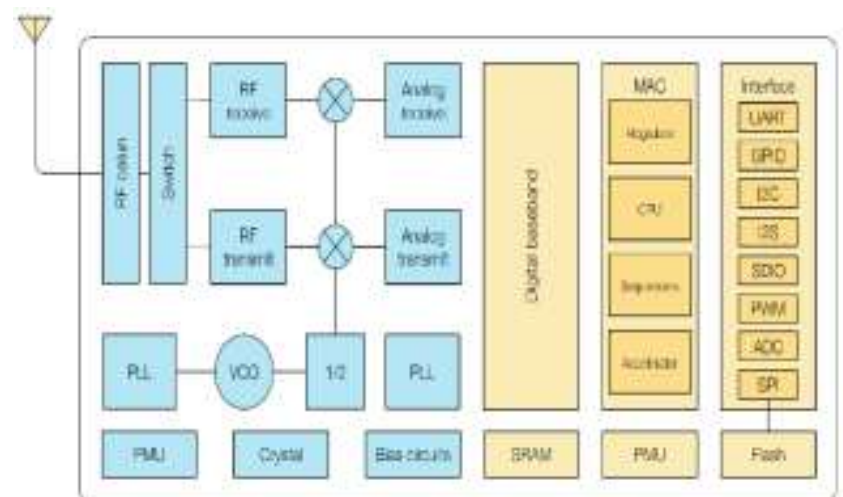

Fig.6. Internal block diagram of ESP8266 module

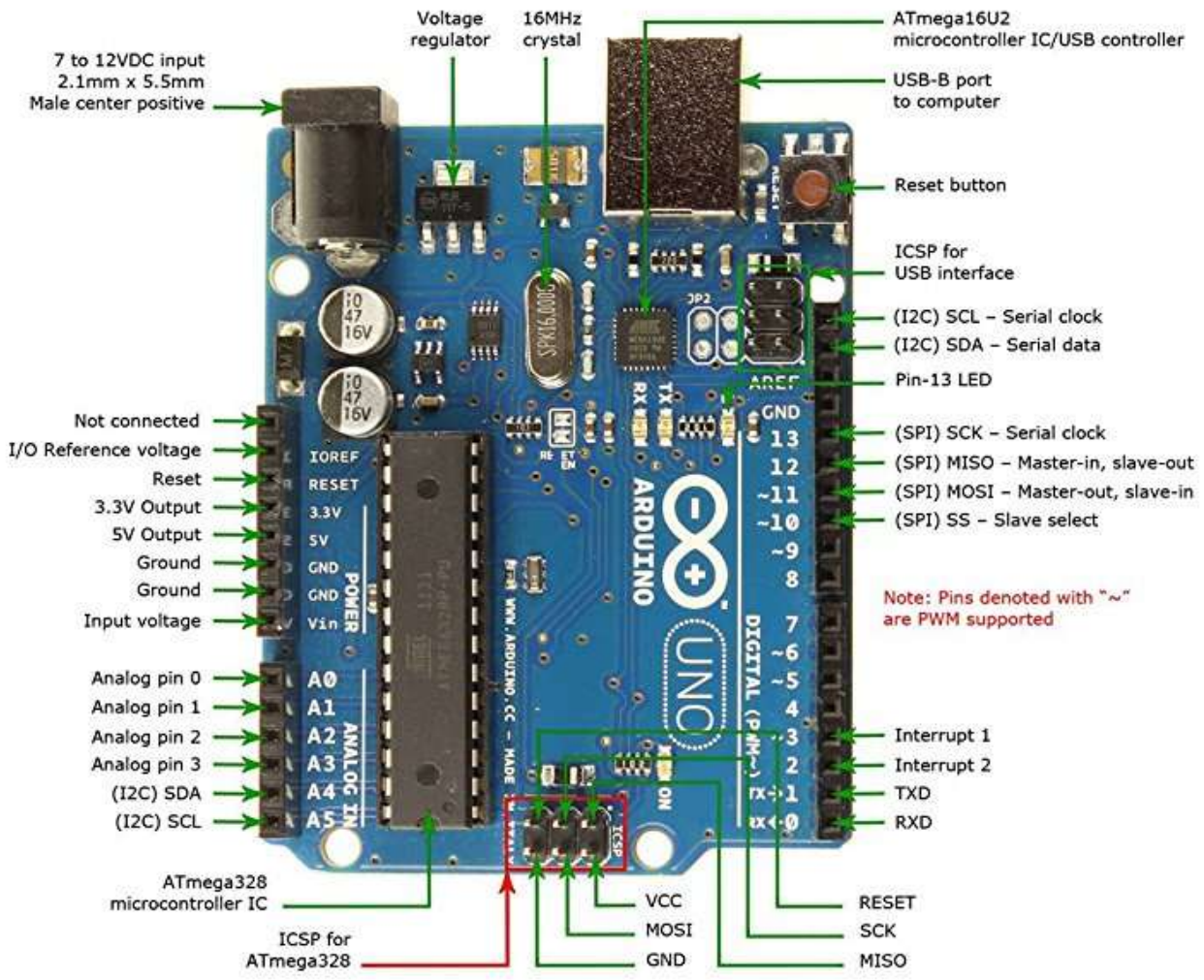

Fig.7. Arduino UNO micro controller

\section{III.METHODOLOGY AND RESULTS OF THE SYSTEM}

The traffic control system operates with 4 different cases which is explained in this section.

\section{Case 1: Normal operating condition}

In the normal operating condition, the fixed delay time is set in the micro controller .So initially one lane will be having green signal and remaining all having red signals. Then after the fixed delay time the next lane alone will be having green signal. This continues for remaining two lanes also.

\section{Case 2: Density based operation}

Eight IR sensors are used in the system. The IR sensors used in this system are emitting IR rays continuously. The vehicles passing the rays reflect the rays and make the IR receiver in the module to receive the reflected signals. This

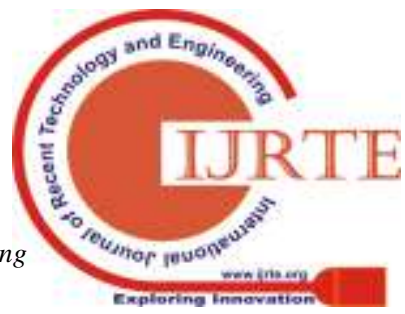




\section{DENSITY CUM PRIORITY BASED ADAPTIVE TRAFFIC LIGHT CONTROL SYSTEM}

makes the comparator in the module to set zero. The comparator output is processed by Arduino UNO micro controller. So if the density in the particular lane is high, then that will be sensed by the IR sensor module. This is processed by the micro controller and if it is beyond the preset limit then the timing for the green signal in the particular lane is increased by the microcontroller.

\section{Case 3: Priority based operation}

The priority vehicle is having the RFID tag, which have the VIN (Vehicle Identification Number) stored in it. The VIN have the information about priority of the vehicle and the type of the vehicle( Ambulance, fire Engine, Police car etc..). So when a priority vehicle is passing the lane then that will be sensed by EM-18 RF reader module and the timing of green signal in the particular lane is increased. The prototype is having only one RFID reader module to explain about the priority operation. In the prototype, that RFID module is connected with right side lane. But the actual system can have 8 RFID reader module to make the system effective.

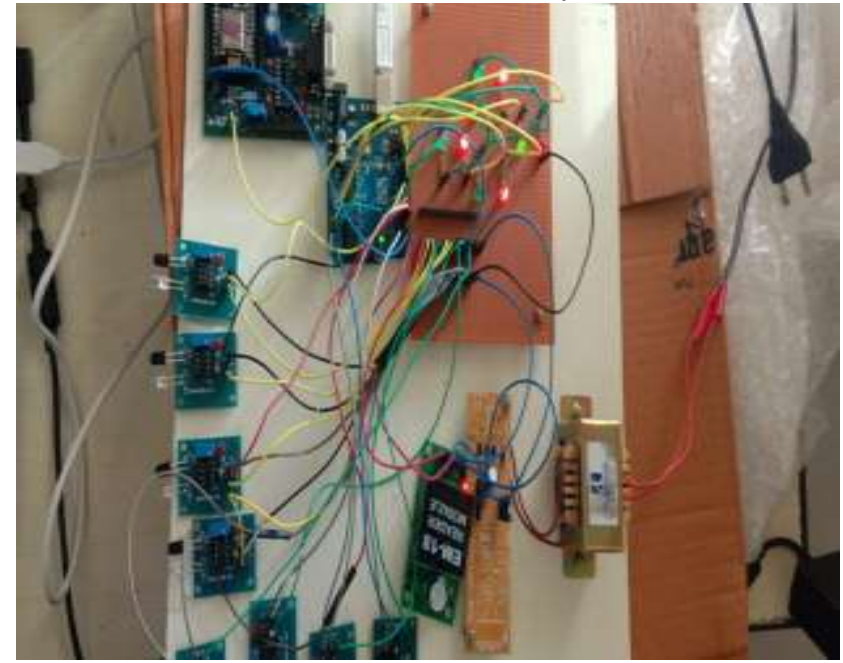

Fig.8.Traffic control system under normal condition with green signal for right side lane

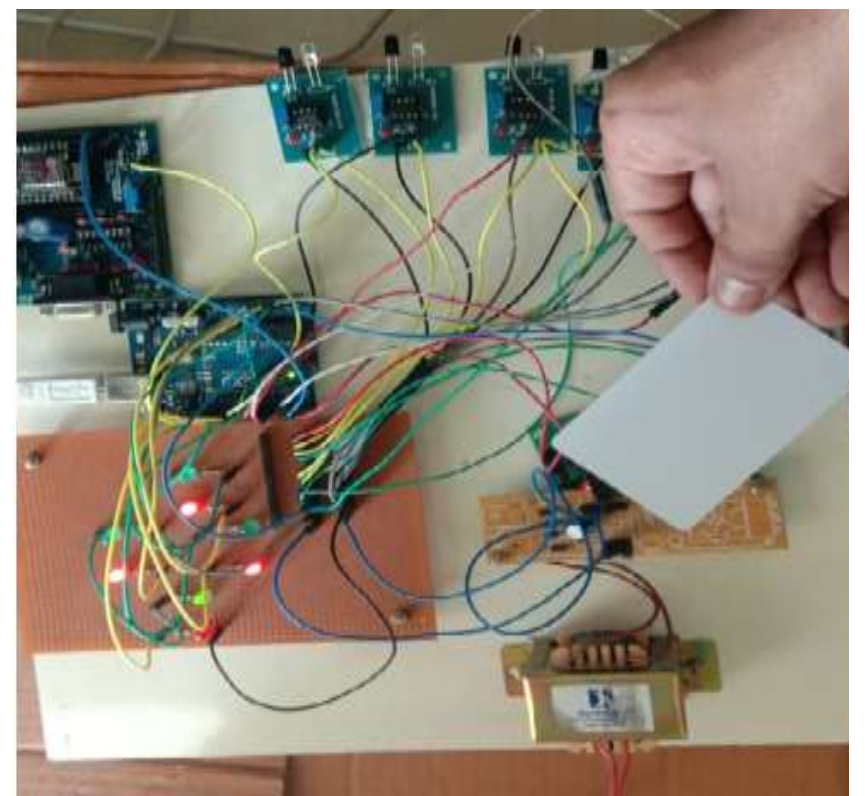

Fig.9.Traffic control system under priority condition with continued green signal for right side lane
The figure 8 shows the traffic control system with the right side lane is given green signal under normal operating condition. The system is having 8 IR sensors, an Arduino UNO micro controller, an RFID reader module , 8 LEDs and an IOT Wifi module which is shown in the figure 8 . The figure 9 shows the operation, when the RFID tag is brought near the RFID reader module. Since the RFID module is connected with the right side lane, the figure 9 shows the right side lane continues with green signal for some more time. The IOT web page in the figure 10 is showing the traffic data with exact timing which can be stored for surveying to design better traffic control system in future.

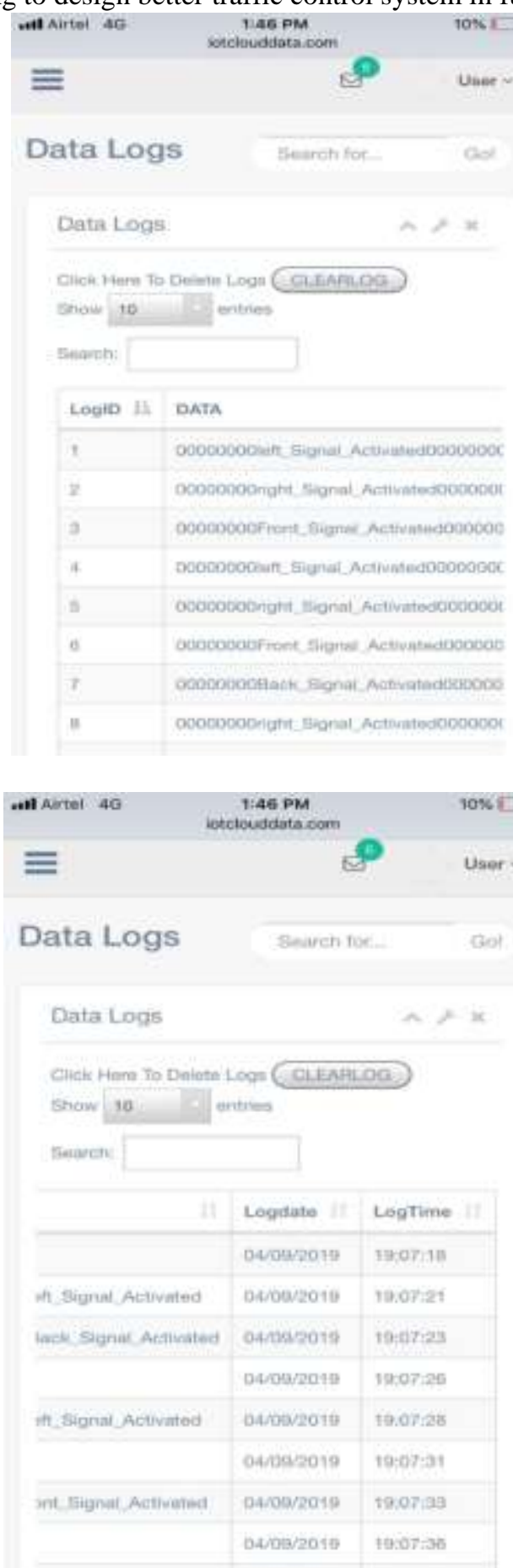

Fig.10 Traffic data with timing in the IOT web page 


\section{CONCLUSION}

The conventional traffic control system is having fixed passing time for each lane and also having predefined sequence of operation. But the traffic light control system developed in this paper is having adaptive passing time for each lane. Using IR sensors the density of each lane is sensed ,if it exceeds beyond the limit, then the passing time of the particular lane is increased. To give the priority for the emergency vehicles RFID reader module is used, which detects the emergency vehicle and then immediately the particular lane is made active with increased passing time also. During normal condition it acts as conventional traffic control system. So this traffic control system avoids the unnecessary waiting of priority vehicles, and since it is considering the density, the unnecessary traffic jam is also reduced which are the short comes in the conventional control system. The traffic data obtained using the IOT web page are also used to design a smart traffic control system in future.

\section{REFERENCES}

1. Knoop, Victor L., Peter F. de Bakker, Christian CJM Tiberius, and Bart van Arem. "Lane determination with GPS precise point positioning." IEEE Transactions on Intelligent Transportation Systems 18, no. 9 (2017): 2503-2513.

2. Gao, Chaoqun, Dongkai Yang, Xuebao Hong, Yao Xu, Bo Wang, and Yunlong Zhu. "Experimental Results About Traffic Flow Detection by Using GPS Reflected Signals." IEEE Journal of Selected Topics in Applied Earth Observations and Remote Sensing 11, no. 12 (2018): 5076-5087.

3. Qi, Liang, MengChu Zhou, and WenJing Luan. "A two-level traffic light control strategy for preventing incident-based urban traffic congestion." IEEE transactions on intelligent transportation systems 19, no. 1 (2018): 13-24.

4. Younes, Maram Bani, and Azzedine Boukerche. "Intelligent traffic light controlling algorithms using vehicular networks." IEEE transactions on vehicular technology 65, no. 8 (2016): 5887-5899.

5. Hou, Yandong, Gaochao Wang, and Yi Zhou. "Virtual-grid based traffic control strategy with multiple intersections collaboration." IEEE Access 6 (2018): 40105-40119.

6. Tahmid, Taqi, and Eklas Hossain. "Density based smart traffic control system using canny edge detection algorithm for congregating traffic information." In 2017 3rd International Conference on Electrical Information and Communication Technology (EICT), pp. 1-5. IEEE, 2017.

7. Hashim, N. M. Z., A. S. Jaafar, N. A. Ali, L. Salahuddin, N. R. Mohamad, and M. A. Ibrahim. "Traffic light control system for emergency vehicles using radio frequency." IOSR Journal of Engineering (IOSRJEN) 3, no. 7 (2013): 43-52.

8. Singh, Harpal, Krishan Kumar, and Harbans Kaur. "Intelligent traffic lights based on RFID." International Journal of Computing \& Business Research (2012): 13.

9. Maqbool, Saima, Ulya Sabeel, Nidhi Chandra, and R. U. A. Bhat. "Smart traffic light control and congestion avoidance system during emergencies using Arduino and Zigbee 802.15. 4." International journal of advanced research in computer science and software engineering 3 , no. 6 (2013): 1801-1808.

10. Chavan, Shilpa S., R. S. Deshpande, and J. G. Rana. "Design of intelligent traffic light controller using embedded system." In 2009 Second International Conference on Emerging Trends in Engineering \& Technology, pp. 1086-1091. IEEE, 2009.

11. Ganiyu, R. A., O. T. Arulogun, and O. O. Okediran. "Development Of A Microcontroller-Based Traffic Light System For Road Intersection Control." International journal of scientific \& technology research 3,no.5 (2014): 209-212.

12. Hussian, Rashid, et al. "WSN applications: Automated intelligent traffic control system using sensors." Int. J. Soft Comput. Eng 3, no.3 (2013): 77-81.

13. Kham, Nang Hom, and Chaw Myat Nwe. "Implementation of modern traffic light control system." International journal of scientific and research publications 4 , no. 6 (2014). 